\section{The American Pediatric Society MEMBERSHIP LIST 1976-1977}

\section{Honorary}

nderson, Henning J., M.D., Forarsvej 42, 2920

Charlottenlund, Copenhagen, Denmark

riztia, Anibal, M.D., Department of Pediatrics

Hospital Luis Calvo MacKenna, Santiago, Chile

urnet, Sir MacFarland, Department of Microbiology, University of Melbourne, Parkville, N.2 Victoria, Australia

haudhuri, Kshirode C., Director, Institute of Child Health, 56-2 Creek Row, Calcutta 14, India

ebre, Professor Robert, 5 Rue de l'Universite. Paris 7E, France

el Mundo, Professor Fe, The Children's Memorial Hospital, 11 Banawe, Quezon City, The Philippines

ogramaci, Professor Ihsan A., Hacettepe Children's Hospital, Ankara, Turkey

poxiadis, Spyros A., M.D., 3, North Vamva Street, Athens 138, Greece

nders, John Franklin, M.D., 300 Longwood Avenue, Boston, Massachusetts 02115

vans, Philip, M.D., Hospital for Sick Children, Great Ormond Street, London WCIN 3JH, England

anconi, Professor Guido, Kinderspital, Zurich, Switzerland
Freud, Anna, 20, Maresfield Gardens, London, N.W. 3 5SX, England

Friderichsen, Professor Carl, Groningen 15, Copenhagen K. Denmark

Gomeż-Risco, Professor Federico, 240 Jardines del Pedregal de San Angel, Mexico, D.F., Mexico

Hubble, D. V., Dean, Yonder Hill, Cold Ash near Newbury, Berkshire, England

Jonxis, Professor J. H. P., Rijkestraatweg 65, Haren Gr., The Netherlands

Karlberg, Petter J. E., M.D., Göteborg University, Vasaparken 411-24, Gothenburg, Sweden

Kerpel-Fronius Professor E., 2nd Department of Pediatrics Medical School of Budapest, Budapest

Lelong, Professor Marcel, 197 Boulevard St. Germain, Paris 7, France

Lightwood, Professor Reginald, Villa 3A, Trebaluger, Menorca, Balearic Isles, Spain

Lind, Professor John, Karolinska Sjukhuset, Stockholm 60, Sweden

McCance, R. A., M.D., Sidney Sussex College, Cambridge, England

Minkowski, Alexandre, M.D., Hôpital Port Royal, IX Tuzolto W.7, Hungary
123, Boulevard de Port-Royal, 75674 Paris Cedex 14, France

Prader, Andrea, M.D., Kinderspital, Zurich, Switzerland

Rossi, Professor Ettore, Universitas-Kinderklinik, Bern, Switzerland

Royer, Pierre, M.D., Professeur Agrege a la Faculte de Medecine, 2, Rue Villersexel, Paris 7EME, France

Scroggie, Arturo, M.D., Hospital Roberto del Rio, Santiago, Chile

Sereni, Professor Fabio, Istituto de Puericultur, Dell Univers. di Milano, Via Commenda 9, Milano 20122, Italy

Tizard, John Peter Mills, M.D., John Radcliffe Hospital, Headington, Oxford, OX3 9DU, England

Vahlquist, Professor Bo, Barnkliniken, Akademiska Sjukhuset, Uppsala, Sweden

Williams, Cicely D., M.D., Tulane University, School of Public Health and Tropical Medicine, 1430 Tulane Avenue, New Orleans, Los Angeles 70112

Wolf, Otto, M.D., Hospital for Sick Children, Great Ormond Street, London W.C.I. England

Ylppo, Professor Arvo, Lasten Klinikka, Helsinki, Finland 